\title{
A microbiological explanation for the obesity pandemic?
}

\author{
Louis Valiquette MD MSC FRCPC ${ }^{1}$, Stéphanie Sirard MSc ${ }^{1}$, Kevin Laupland MD MSC FRCPC ${ }^{2,3}$
}

$\mathrm{T}$ he prevalence of obesity is increasing worldwide. According to the Canadian Health Measures Survey, in 2011, one in four Canadians was obese ( $25 \%$ of women; $27 \%$ of men) (1). In addition to the imbalance between energy intake and expenditure, sedentary lifestyle, a diet high in saturated fats and sugars, and genetic predisposition, many other factors may be involved in obesity. The presence of either symbiotic or pathogenic microorganisms may contribute to the development of obesity. With the progress of metagenomics and molecular techniques in recent years was born an interest in the microorganisms living on and inside us (2). More than $10^{14}$ microorganisms, which represent up to 1150 different species and a total genome comprising 150 -fold more genes than the human genome, live in our gastrointestinal tracts (3). The gut microbiota is known to play a role in protection against pathogenic bacteria, immune function and digestion by degrading nondigestible carbohydrates such as cellulose, pectin and starch $(4,5)$. Over the past decade, several prominent publications have led to an intriguing hypothesis that links differences in gut microbial ecology to energy homeostasis. In simpler words, some individuals harbouring a microbiota more efficient at extracting energy from the diet (eg, through the ability to degrade indigestible components) may be at higher risk for obesity. This hypothesis is interesting but controversial, and raises several questions.

The bacterial phyla Firmicutes (which include genera Clostridium, Ruminococcus and Lactobacillus) and Bacteroidetes (including genera Prevotella and Bacteroides) account for $90 \%$ of the gut microbiota (6). The balance of these populations is essential for the maintenance of a healthy microbiota. The microbiota of genetically obese mice was associated with a $50 \%$ reduction in Bacteroidetes and a proportional increase in Firmicutes compared with lean animals (7). An interesting observation was made when colonizing germ-free mice with the gut microbiota of normal mice. This transplantation led to a $60 \%$ increase in body fat and insulin resistance within two weeks, in spite of reduced food consumption (8).

Findings about the implication of the proportion of Firmicutes and Bacteroidetes in human obesity are contradictory because some studies have found no difference in the Firmicutes and Bacteroidetes ratio $(9,10)$, while others have (11). A diet-induced weight loss in obese individuals was associated with a reduction in the Firmicutes: Bacteroidetes ratio, which was similar to the lean controls (11). Recently, a decrease in specific species (Bifidobacterium animalis and Methanobrevibacter smithii) and an increase in others, such as Staphylococcus aureus, Escherichia coli and Lactobacillus reuteri, have been associated with obesity (12). Normal-weight children had greater bifidobacteria counts and fewer fecal numbers of $S$ aureus compared with overweight/obese children (13). These findings propose that gut microbiota in infancy may predict obesity, reinforcing the importance of prevention. Conflicting findings provide evidence of variability among individuals and in one individual over time. Some changes in gut microbiota may influence individuals to a different extent, and more studies involving large obese populations are required to draw more precise conclusions.

Although their exact contribution remains unclear, microorganisms in the gut are believed to be involved in the development of obesity by two different and complementary mechanisms. They can extract energy from nondigestible polysaccharides and produce low-grade inflammation (14). Because many Firmicutes are major butyrate producers, an abundance of bacteria from this phylum could be associated with an increase in genes encoding enzymes that enable the degradation of complex polysaccharides and, in turn, increase the production of monosaccharides and short-chain fatty acids (SCFAs) (15). Up to $10 \%$ of the total energy extracted from food corresponds to SCFA production (10). In a mouse model, the obese microbiome was found to be richer in enzymes involved in the digestion of complex polysaccharides. Consequently, higher concentrations of butyrate and acetate were found in these mice cecum (16). This increased capacity for extracting energy was also transferred to germ-free mice when they were colonized with an obese microbiota (16). In a cross-sectional study, overweight and obese individuals also exhibited higher fecal levels of end products of colonic fermentation (butyrate, acetate and SCFAs) than normal-weight individuals, thus suggesting a more efficient energy extraction process (10).

Obesity has been associated with chronic low-grade inflammation; however, how they are linked remains unclear (17). The potential implication of bacterial lipopolysaccharide (LPS) in obesity was highlighted in a mouse model. Interestingly, food was found to modulate plasma LPS levels (endotoxemia) and these levels were related to fat content. In addition, these concentrations were sufficient to induce the development of metabolic disorders (essentially, diabetes and obesity) (18). Later, an association was reported between endotoxemia and energy intake in humans, but not between LPS and weight or body mass index (19). More studies are needed to elucidate the role of endotoxemia in human obesity. Over the past several years, studies investigating gut microbiota and obesity have accumulated. Even in the absence of consensus, researchers agree that there is undoubtedly a link between gut microbiota and metabolic diseases such as obesity.

In addition to the interaction between microbiota and obesity, some studies have also attempted to show a direct relationship with some pathogenic microorganisms. The concept of 'infectobesity' (obesity of infectious origin) has recently become more popular. Nevertheless, over the past 30 years, approximately 10 microorganisms (including canine distemper virus, avian adenovirus [SMAM-1] and human adenoviruses) have been linked with obesity in either humans or animals (20). Currently, studies are focusing on adenoviruses, especially adenovirus 36 ( $\mathrm{Ad} 36$ ), which is associated with adiposity and inflammation. Although Ad36 has been reported to induce obesity in animal models $(21,22)$, results have been contradictory in humans. A greater prevalence of neutralizing antibodies was observed in obese individuals compared with nonobese individuals in both adults (23-25) and children (26-29). However, some studies did not achieve the same results in adults (30-32) or children (33). In a meta-analysis of 10 studies (34), Ad36 infection was associated with an increased risk for obesity (OR 1.9 [95\% CI 1.01 to 3.56]; P=0.047) and weight gain (increase in body mass index of $3.19 \mathrm{~kg} / \mathrm{m}^{2}$ ). These variable results are questionable because positive associations were essentially reported by a limited group of authors from similar institutions and because Ad36 was identified using serology in these studies. Discrepancies in detection and specificity

${ }^{1}$ Department of Microbiology-Infectious Diseases, Université de Sherbrooke, Sherbrooke, Quebec; ${ }^{2}$ Department of Medicine, Royal Inland

Hospital, Kamloops, British Columbia; ${ }^{3}$ Departments of Medicine, Critical Care Medicine, Pathology and Laboratory Medicine, and

Community Health Sciences, University of Calgary, Calgary, Alberta 
may exist between studies using serology and those using viral culture or polymerase chain reaction detection (35). Moreover, conclusions originate from observational studies; thus, a causal relation between Ad36 infection and obesity cannot be proven. In addition, studies appear to be more consistent in children than in adults, likely indicating differences in the time the infection occurred or in viral load. Ad36 antibodies were also detected in nonobese individuals, reinforcing the contribution of other factors to the development of obesity and calling into question the implication of this particular adenovirus in obesity.

A potential vaccine for prophylactic treatment of Ad36-induced obesity was evaluated in a mouse model (36). A greater body weight in the control group and decreased levels of proinflammatory cytokines in the vaccinated mice were observed. These results are promising, although more studies targeting other adenoviruses are needed because the relevance and applicability in humans is unknown.

Interindividual variation in microorganisms makes drawing conclusions more complex, but gut microbiota deserves more attention as a potential target in prevention or control of obesity. Personalized therapy based on the microbiome may be the key to overcome this variation. Modulating gut microbiota by the consumption of beneficial

\section{REFERENCES}

1. Statistics Canada. 2013. Body composition of Canadian adults, 2009 to 2011. <www.statcan.gc.ca/pub/82-625-x/2012001/ article/11708-eng.htm > (Accessed October 2014).

2. Culligan EP, Sleator RD, Marchesi JR, Hill C. Metagenomics and novel gene discovery: Promise and potential for novel therapeutics. Virulence 2014;5:399-412.

3. Qin J, Li R, Raes J, et al. A human gut microbial gene catalogue established by metagenomic sequencing. Nature 2010;464:59-65.

4. Hooper LV, Midtvedt T, Gordon JI. How host-microbial interactions shape the nutrient environment of the mammalian intestine. Ann Rev Nutr 2002;22:283-307.

5. Zoetendal EG, Vaughan EE, de Vos WM. A microbial world within us. Mol Microbiol 2006;59:1639-50.

6. Gangarapu V, Yıldız K, Ince AT, Baysal B. Role of gut microbiota: Obesity and NAFLD. Turk J Gastroenterol 2014;25:133-40.

7. Ley RE, Bäckhed F, Turnbaugh P, et al. Obesity alters gut microbial ecology. Proc Natl Acad Sci U S A 2005;102:11070-5.

8. Bäckhed F, Ding H, Wang T, et al. The gut microbiota as an environmental factor that regulates fat storage. Proc Natl Acad Sci U S A 2004;101:15718-23.

9. Moreno-Indias I, Cardona F, Tinahones FJ, Queipo-Ortuño MI. Impact of the gut microbiota on the development of obesity and type 2 diabetes mellitus. Front Microbiol 2014;5:190.

10. Fernandes J, Su W, Rahat-Rozenbloom S, Wolever TM, Comelli EM. Adiposity, gut microbiota and faecal short chain fatty acids are linked in adult humans. Nutr Diabetes 2014;4:e121.

11. Ley RE, Turnbaugh PJ, Klein S, Gordon JI. Microbial ecology: Human gut microbes associated with obesity. Nature 2006;444:1022-3.

12. Million M, Maraninchi M, Henry M, et al. Obesity-associated gut microbiota is enriched in Lactobacillus reuteri and depleted in Bifidobacterium animalis and Methanobrevibacter smithii. Int J Obes (Lond) 2012;36:817-25.

13. Kalliomäki M, Collado MC, Salminen S, Isolauri E. Early differences in fecal microbiota composition in children may predict overweight. Am J Clin Nutr 2008;87:534-8.

14. Doré J, Simrén M, Buttle L, Guarner F. Hot topics in gut microbiota. United European Gastroenterol J 2013;1:311-8.

15. Duca FA, Lam TK. Gut microbiota, nutrient sensing and energy balance. Diabetes Obes Metab 2014;16(Suppl 1):68-76.

16. Turnbaugh PJ, Ley RE, Mahowald MA, et al. An obesity-associated gut microbiome with increased capacity for energy harvest. Nature 2006;444:1027-31.

17. Lin CS, Chang CJ, Lu CC, et al. Impact of the gut microbiota, prebiotics, and probiotics on human health and disease. Biomed J 2014;37:259-68.

18. Cani PD, Amar J, Iglesias MA, et al. Metabolic endotoxemia initiates obesity and insulin resistance. Diabetes 2007;56:1761-72.

19. Amar J, Burcelin R, Ruidavets JB, et al. Energy intake is associated with endotoxemia in apparently healthy men. Am J Clin Nutr 2008;87:1219-23. bacteria or the use of prebiotics or drugs targeting specific bacterial populations could be an interesting avenue in the treatment of obesity. Fecal microbiota transplantation (FMT) could also represent a potential therapy against obesity and metabolic diseases; however, given all of the hurdles that are met by physicians willing to proceed with FMT for recurrent $\mathrm{C}$ difficile infection, even while supported by strong evidence, we are still far from using FMT to treat other conditions. At the time the present article was written, no clinical trial was registered on www.clinicaltrials.gov on the effect of FMT on obesity. The hypothesis of a single agent as a cause or a potentiator of obesity is less robust for several reasons: the evidence is clearly not as strong as for the microbiota hypothesis; it comes mainly from the same research team; and accepting this hypothesis would mean that obesity is transmissible, which makes little sense in our opinion.

Although the primary cause of obesity will always be a misbalance between energy intake and expenditure, its association with microbiology is only a small demonstration of how complex this multifactorial condition is. Nevertheless, beneficial as well as pathogenic microorganisms deserve more attention as evidence of their contribution to metabolic disorders continues to accumulate.

20. Dhurandhar NV. A framework for identification of infections that contribute to human obesity. Lancet Infect Dis 2011;11:963-9.

21. Dhurandhar NV, Whigham LD, Abbott DH, et al. Human adenovirus Ad-36 promotes weight gain in male rhesus and marmoset monkeys. J Nutr 2002;132:3155-60.

22. Pasarica M, Shin AC, Yu M, et al. Human adenovirus 36 induces adiposity, increases insulin sensitivity, and alters hypothalamic monoamines inrats. Obesity (Silver Spring) 2006;14:1905-13.

23. Atkinson RL, Dhurandhar NV, Allison DB, et al. Human adenovirus-36 is associated with increased body weight and paradoxical reduction of serum lipids. Int J Obes (Lond) 2005;29:281-6.

24. Trovato GM, Castro A, Tonzuso A, et al. Human obesity relationship with Ad36 adenovirus and insulin resistance. Int J Obes (Lond) 2009;33:1402-9.

25. Trovato GM, Martines GF, Garozzo A, et al. Ad36 adipogenic adenovirus in human non-alcoholic fatty liver disease. Liver Int 2010;30:184-90.

26. Atkinson RL, Lee I, Shin HJ, He J. Human adenovirus-36 antibody status is associated with obesity in children. Int J Pediatr Obes 2010;5:157-60.

27. Na HN, Hong YM, Kim J, Kim HK, Jo I, Nam JH. Association between human adenovirus-36 and lipid disorders in Korean schoolchildren. Int J Obes (Lond) 2010;34:89-93.

28. Gabbert C, Donohue M, Arnold J, Schwimmer JB. Adenovirus 36 and obesity in children and adolescents. Pediatrics 2010;126:721-6.

29. Cakmakliogullari EK, Sanlidag T, Ersoy B, et al. Are human adenovirus -5 and 36 associated with obesity in children? J Investig Med 2014;62:821-4.

30. Broderick MP, Hansen CJ, Irvine M, et al. Adenovirus 36 seropositivity is strongly associated with race and gender, but not obesity, among US military personnel. Int J Obes (Lond) 2010;34:302-8

31. Goossens VJ, deJager SA, Grauls GE, et al. Lack of evidence for the role of human adenovirus-36 in obesity in a European cohort. Obesity (Silver Spring) 2011;19:220-1.

32. Na HN, Kim J, Lee HS, et al. Association of human adenovirus-36 in overweight Korean adults. Int J Obes (Lond) 2012;36:281-5.

33. Berger PK, Pollock NK, Laing EM, et al. Association of adenovirus 36 infection with adiposity and inflammatory-related markers in children. J Clin Endocrinol Metab 2014;99:3240-6.

34. Yamada T, Hara K, Kadowaki T. Association of adenovirus 36 infection with obesity and metabolic markers in humans: A meta-analysis of observational studies. PLoS One 2012;7:e42031.

35. Goossens VJ. Adenovirus-36 prevalence paradox in development. J Clin Virol 2013;56:5-6.

36. Na HN, Nam JH. Proof-of-concept for a virus-induced obesity vaccine; vaccination against the obesity agent adenovirus 36 . Int J Obes (Lond) 2014 [Epub ahead of print]. 


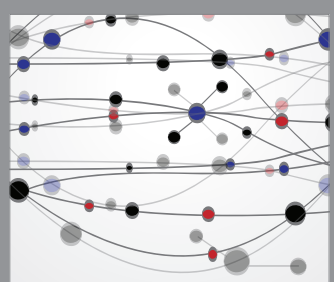

The Scientific World Journal
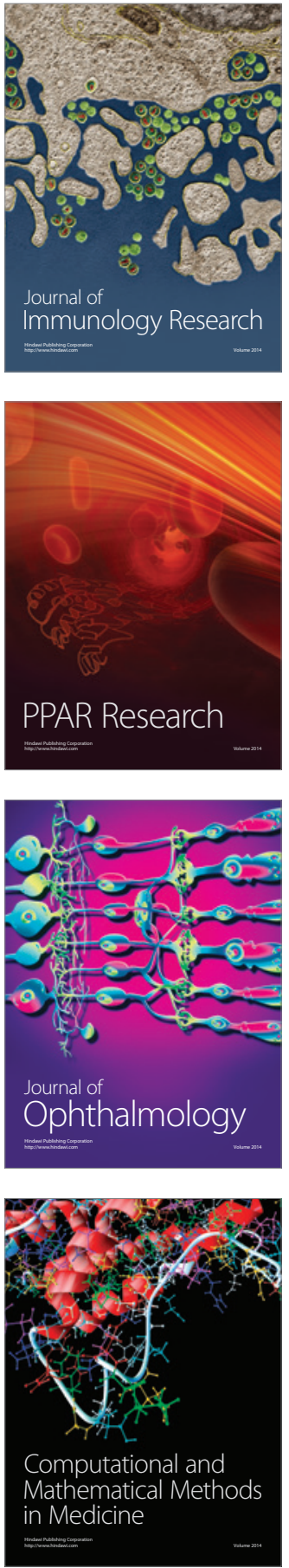

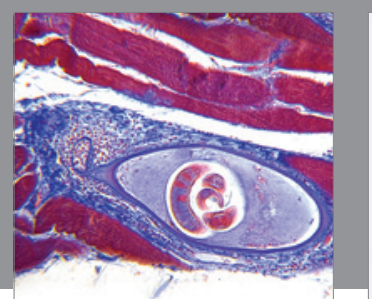

Gastroenterology Research and Practice

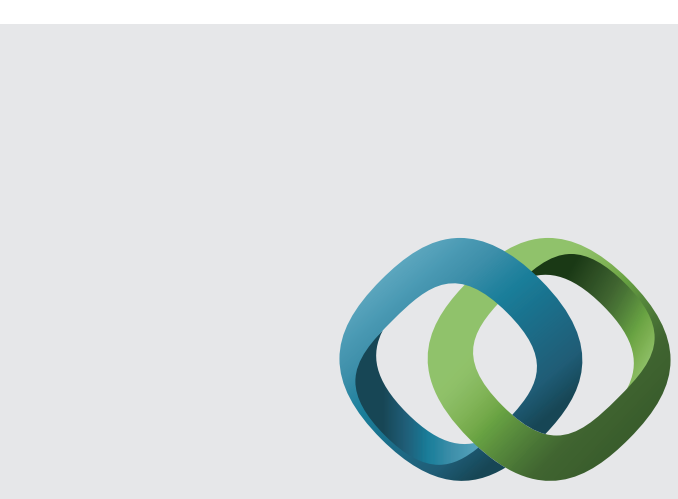

\section{Hindawi}

Submit your manuscripts at

http://www.hindawi.com
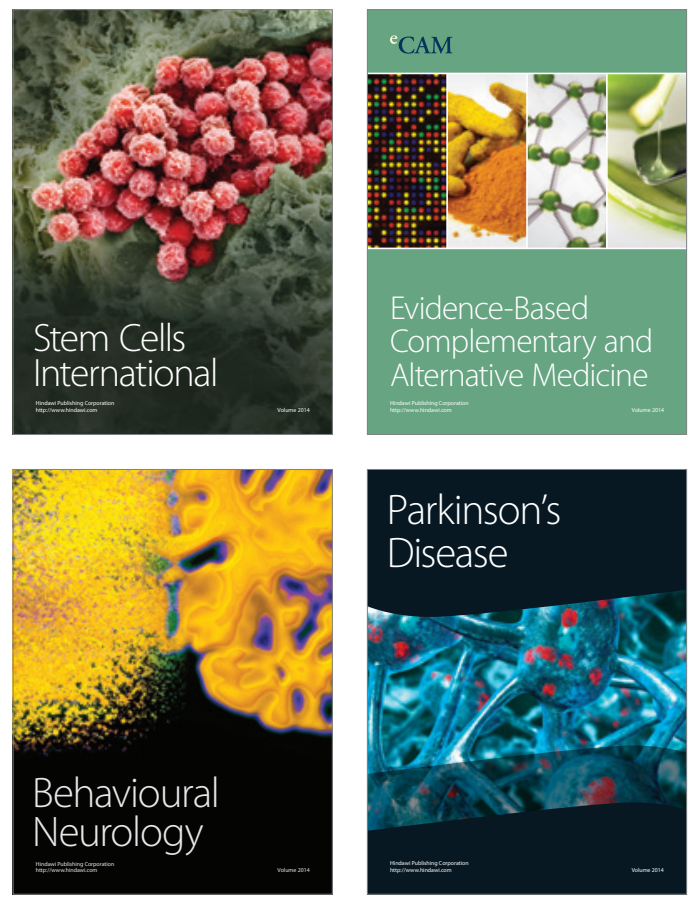
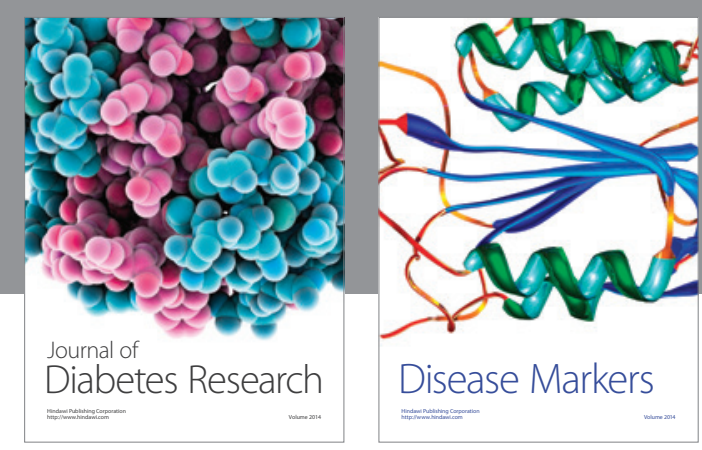

Disease Markers
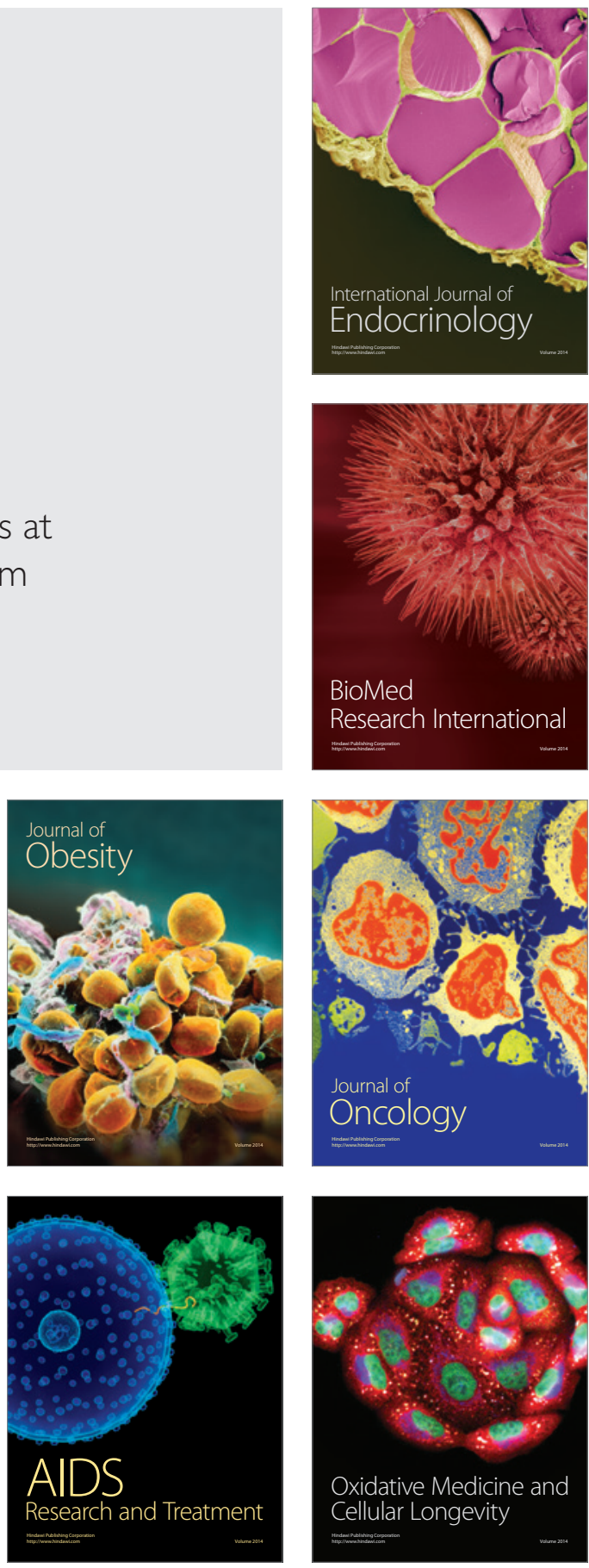